\title{
Correlation between Estradiol Levels and Insulin Resistance in Patients with Polycystic Ovary Syndrome
}

\section{ART ICLE INFO}

\section{Article Type}

Descriptive Study

\section{Authors}

Khosromehr M.* MD,

Alirezaei M. ${ }^{1} M D$
How to cite this article Khosromehr M, Alirezaei M. Correlation between Estradiol Levels and Insulin Resistance in Patients with Polycystic Ovary Syndrome. Sarem Journal of Reproductive Medicine. 2019;3(4):149-154

\section{A B S T R A C T}

Aims Polycystic Ovary Syndrome (PCOS) causes metabolic disorders and endocrine disruptions in the body. Serum levels of some hormones in this disease are abnormal, including increased levels of estradiol in the blood. On the other hand, women with PCOS have insulin resistance. The aim of this study was to evaluate the correlation between estradiol and insulin levels in PCOS patients referred to Sarem Hospital.

Instrument \& Methods This descriptive cross-sectional study was performed on 567 patients with PCOS referring to the infertility clinic of Sarem Hospital. Demographic and disease information was collected through a questionnaire and the results were analyzed by SPSS 22 software.

Findings There was a significant correlation between BMI and fasting ( $\mathrm{p}=0.0001)$ and $2 \mathrm{hpp}$ $(p=0.001)$ insulin. Also a weak and inverse correlation was observed between fasting insulin and prolactin levels $(\mathrm{p}=0.005)$. There was a weak correlation between fasting $(\mathrm{p}=0.004)$ and $2 \mathrm{hpp}(\mathrm{p}=0.0001)$ insulin levels and menstrual cycle. There was no statistically significant correlation between serum estradiol levels and fasting and $2 \mathrm{hpp}$ insulin ( $\mathrm{p}>0.05)$.

Conclusion There is no correlation between blood estradiol levels and insulin resistance and increased fasting and $2 \mathrm{hpp}$ blood insulin levels in patients with PCOS that lead to insulin resistance in these individuals cannot be used alone as a criterion of PCOS.

Keywords Polycystic Ovary Syndrome; Insulin Resistance; Estradiol
*Sarem Fertility \& Infertility Research Center (SAFIR), Iran University of Medical Sciences, Tehran, Iran ${ }^{1}$ Sarem Fertility \& Infertility Research Center (SAFIR), Iran University of Medical Sciences, Tehran, Iran

\section{* Correspondence}

Address: Sarem Women Hospital, Basij Square, Phase 3, Ekbatan Town, Tehran, Iran. Postal Code: 1396956111

Phone: +98 (21) 44670888

Fax: $+98(21) 44670432$

m_khosromehr@yahoo.com

\section{Article History}

Received: February 24, 2019

Accepted: July 25, 2019

ePublished: October 15, 2019

\section{I T A T I O N L I N K S}

[1] Evaluation of serum prolactin levels in patients with ... [2] Polycystic ovary ... [3] Polycystic ovary syndrome: Important underrecognised ... [4] Lifecycle of polycystic ovary syndrome ... [5] Epidemiology, diagnosis, and management of polycystic ... [6] Diagnosis and treatment of polycystic ovary syndrome: an ... [7] New diagnosis of polycystic ovary ... [8] Polycystic ovary syndrome: Insight into pathogenesis and a ... [9] Effect of Vitex agnus-castus fruits hydroalcoholic ... [10] Current perspectives in polycystic ovary ... [11] An algorithm for treatment of infertile women ... [12] Polycystic Ovary ... [13] Effect of licorice root hydroalcoholic extract ... [14] american association of clinical endocrinologists, American ... [15] The effect of green tea extract on reproductive ... [16] Contemporary and traditional perspectives of ... [17] International evidence-based guideline for the ... [18] The diagnosis of polycystic ovary ... [19] Effects of chamomile extract on biochemical and clinical ... [20] The effect of different intensities of aerobic ... [21] The impact of ethnicity on the presentation ... [22] Racial influence on the polycystic ovary syndrome ... [23] Efficacy of combined metformin-letrozole in comparison ... [24] Circulating prolactin associates with diabetes ... [25] Polycystic ovarian disease in the females 15-45 years ... [26] Reproductive ... [27] Review of Clinical Manifestations and Long ... [28] Effectof estrogen replacement therapy ... [29] Relationship between insulin and ... [30] Associations ofestrogen and testosterone with insulin resistance ... [31] Study of insulin resistance in subclinical ... [32] Longitudinal study of insulin resistance and sex hormones overthe ... [33] Alteration of insulin sensitivity by sex hormones ... 
برخى از هورمونها غيرطبيعى است كه مىتوان به افزايش نسبت

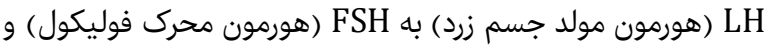

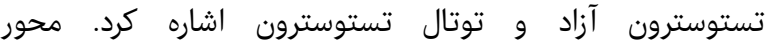

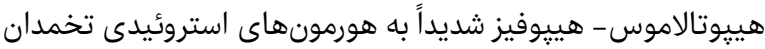

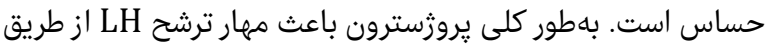

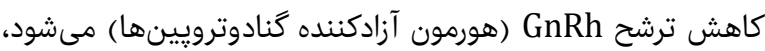

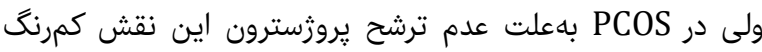

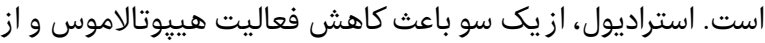

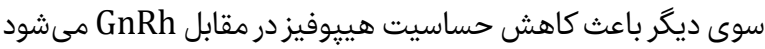

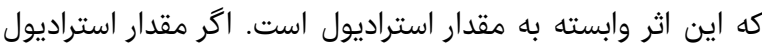

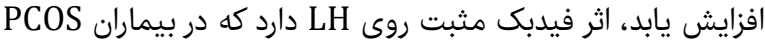

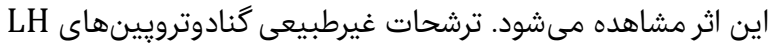

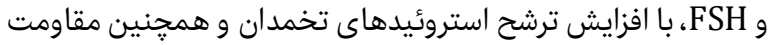

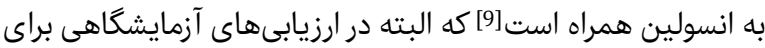

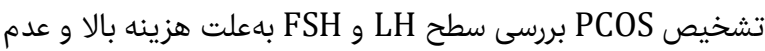

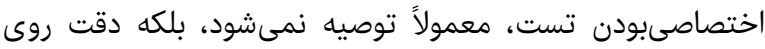

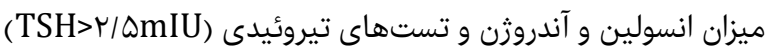

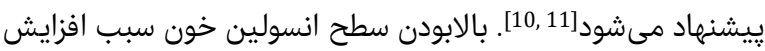

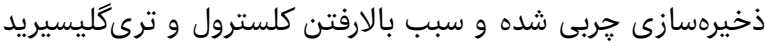

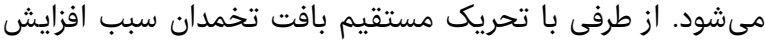

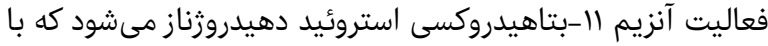

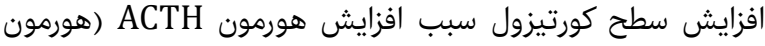

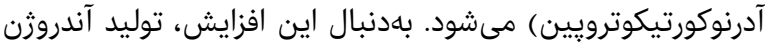

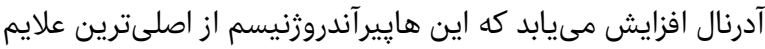

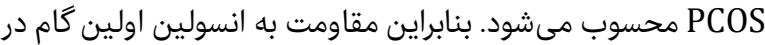

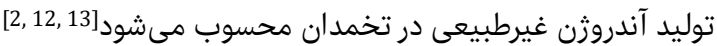

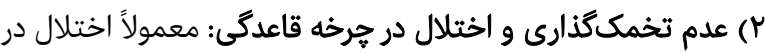

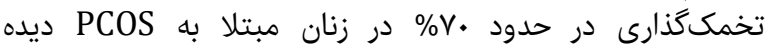

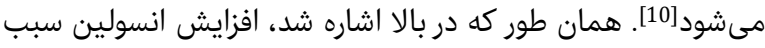

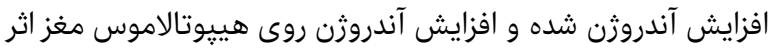

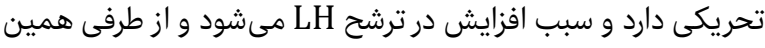

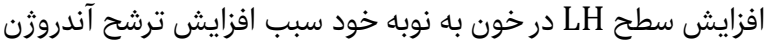

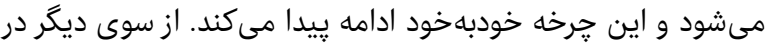

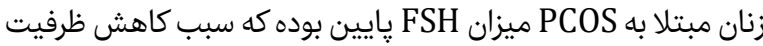

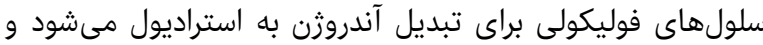

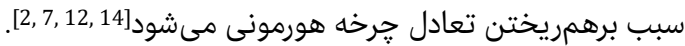

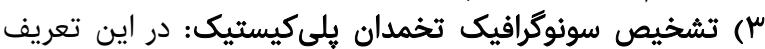

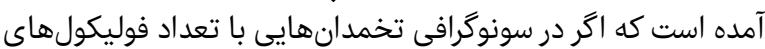

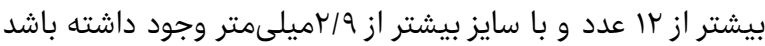

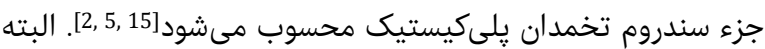

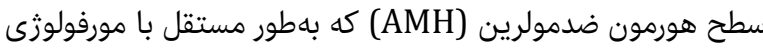

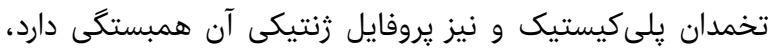

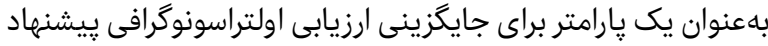

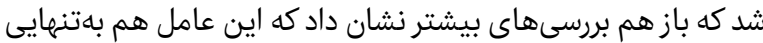

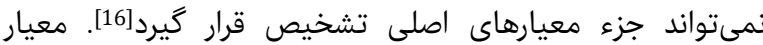

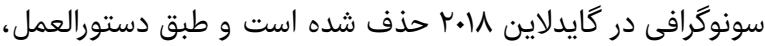

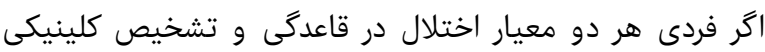

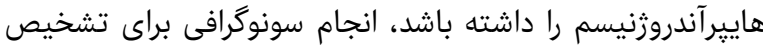

توصيه نمىشود[17].

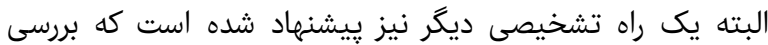

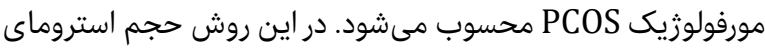

همبستگى ميزان استراديول و مقاومت به انسولين

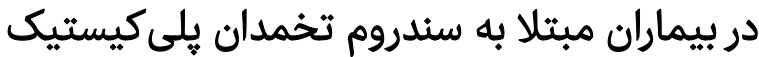

MD " مريم خسرومهر

مركز تحقيقات بارورى و نابارورى صارم، دانشكاه علوم يزشكى ايران، تهران، ايران

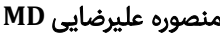
مركز تحقيقات بارورى و نابارورى صارم، دانشگاه علوم يزشكى ايران، تهران، ايران

جكيده اهداف: سندروم تخمدان يلىكيستيك (PCOS) سبب اختلالهاى متابوليك و

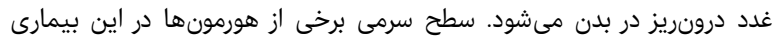

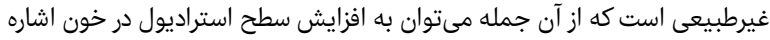

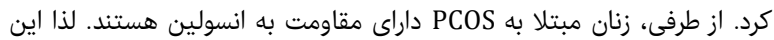

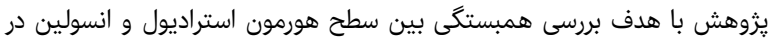

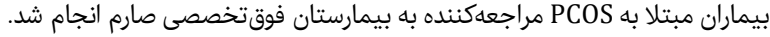

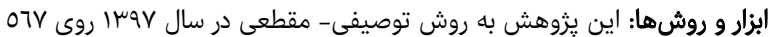

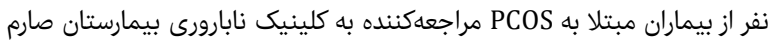

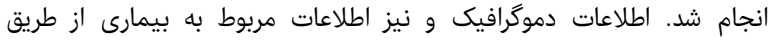

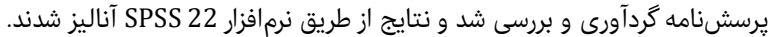

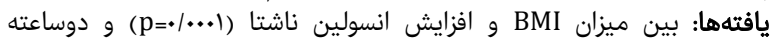

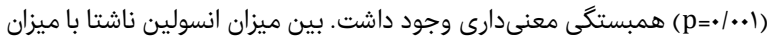

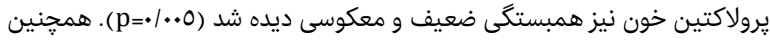

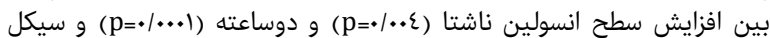

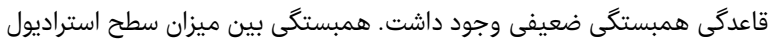

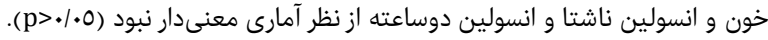

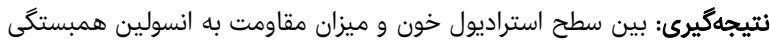

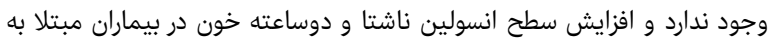
PCOS

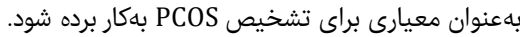
كليدوازمها: سندروم تخمدان بِلىكيستيك، مقاومت به انسولين، استراديول

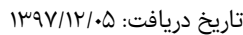

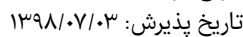

m_khosromehr@yahoo.com : نويسنده مسئول

مقدمه

سندروم تخمدان يلىكيستيك (PCOS) جزء اختلال هاى شايع غدد

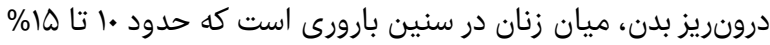

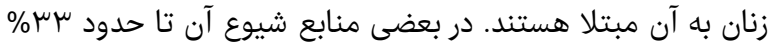

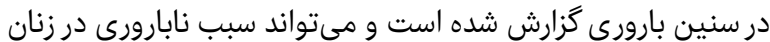

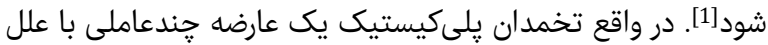

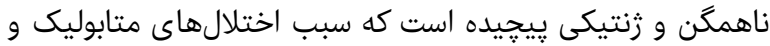

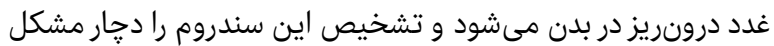

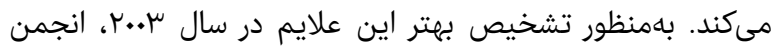

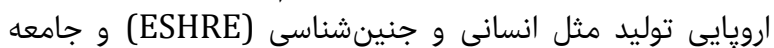

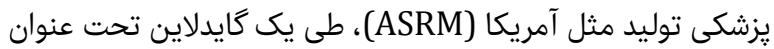

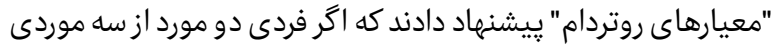

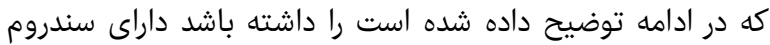

PCOS

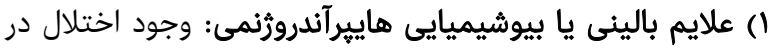

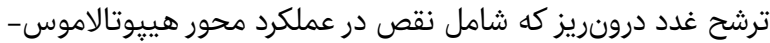

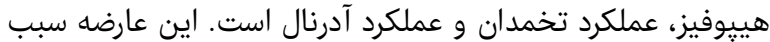

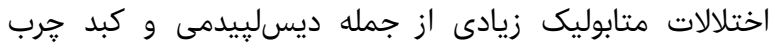

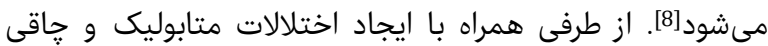

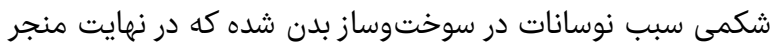

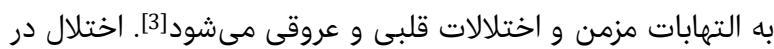

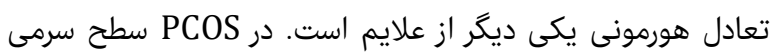




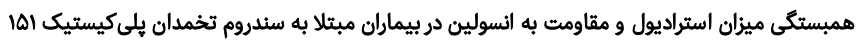

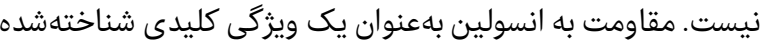

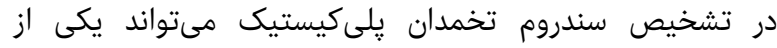
معيارهاى اصلى محسوب شود [117].

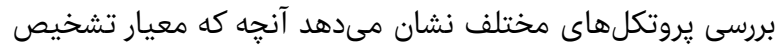

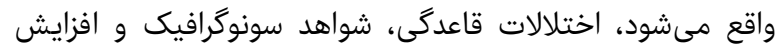

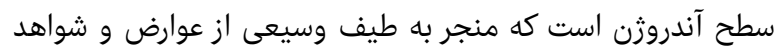

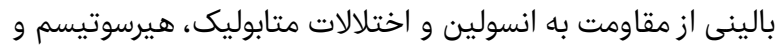

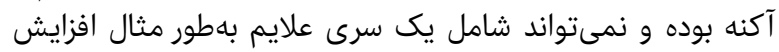

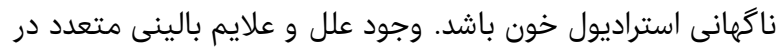

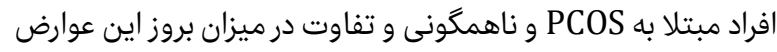

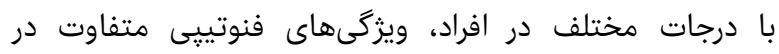

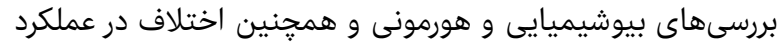

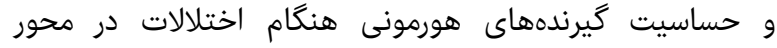

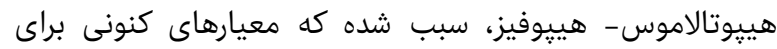

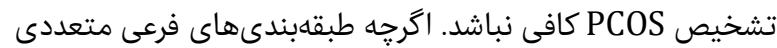

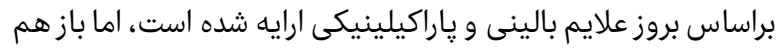

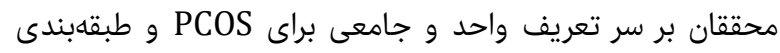

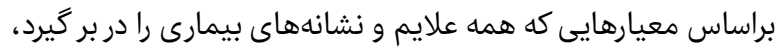

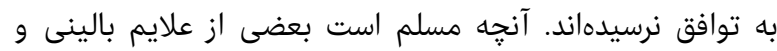

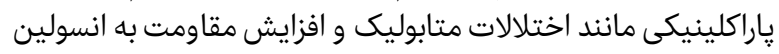

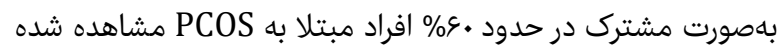

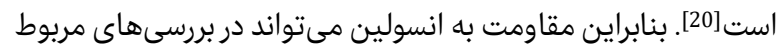

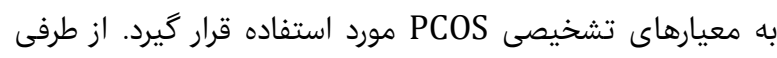

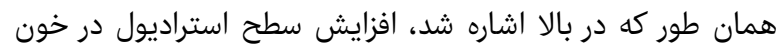

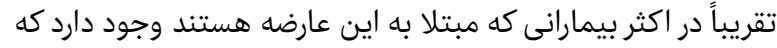

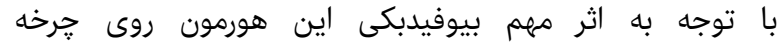

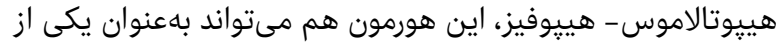
معيارهاى تشخيص بررسى شود. لذا با توجه به اين كه مقاومت تشيص به انسولين يكى ازئ از علايم بالينى شايع

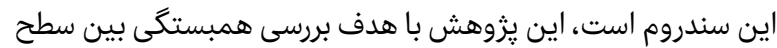

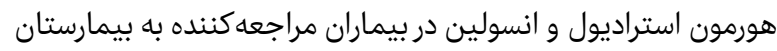
فوقتخصصى صارم انجام شد.

\section{ابزار و روشها}

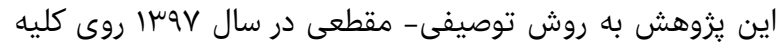

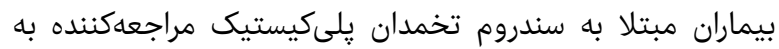

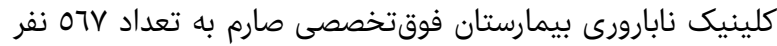

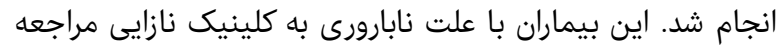

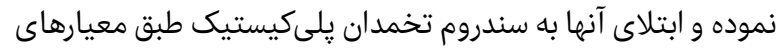

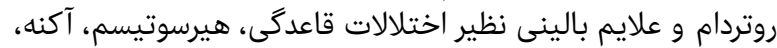

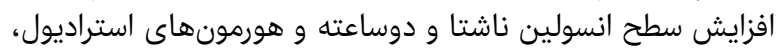

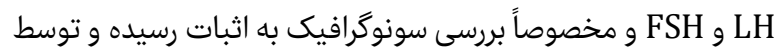

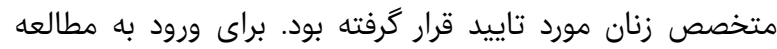

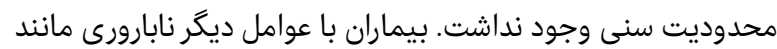

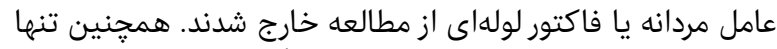

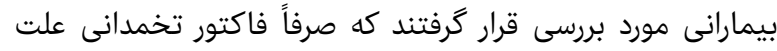

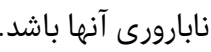

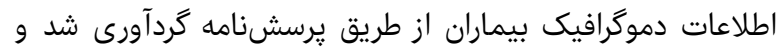

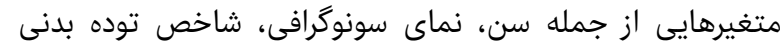

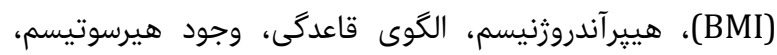

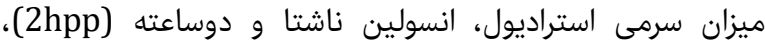

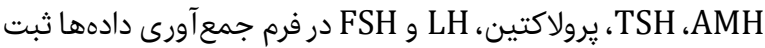

تخمدان نسبت به سطح ارزيابى مىشود (نسبت S/A) مئ اين نسبت

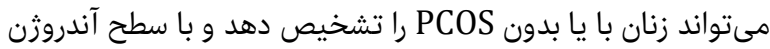

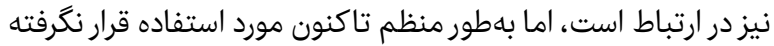

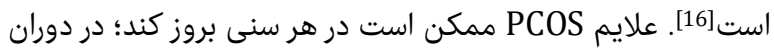

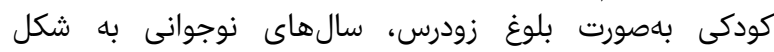

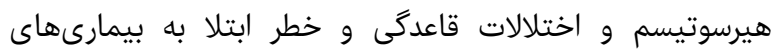

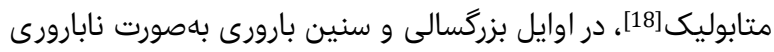

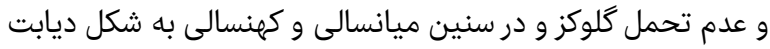

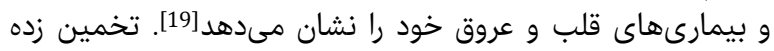

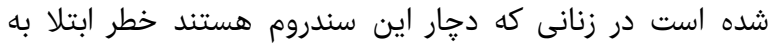

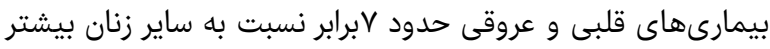

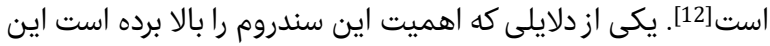

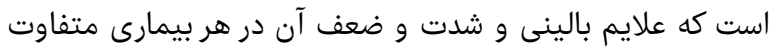

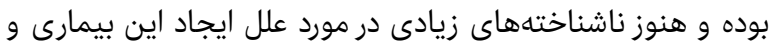

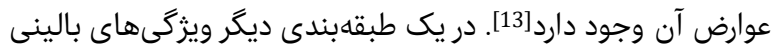

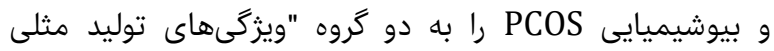

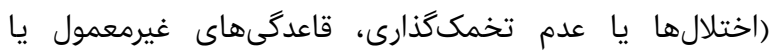

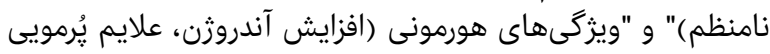

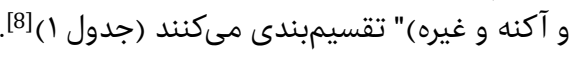

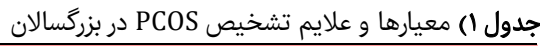

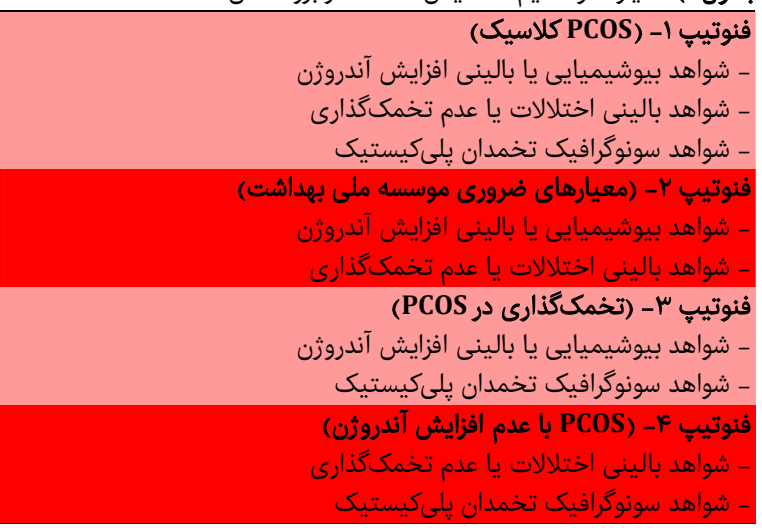

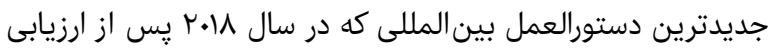

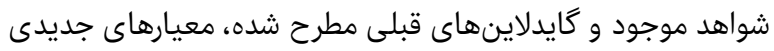

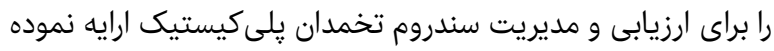

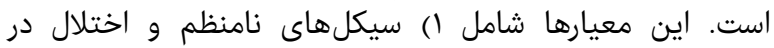

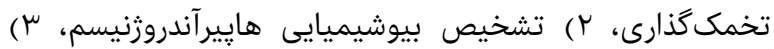

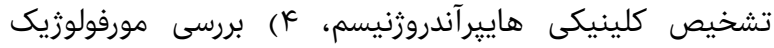

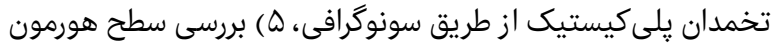

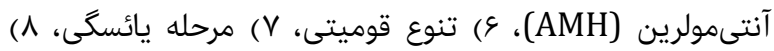

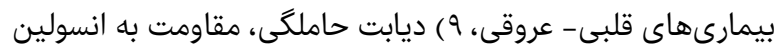

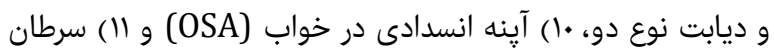
آندومتر مى شوند. بهطور خلاصه مىتوان گفتت بررسى افزايش سطح آندروثن خون از

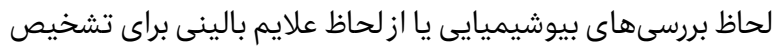

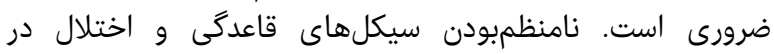

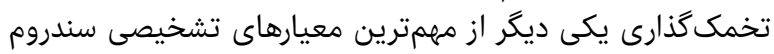

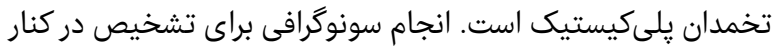

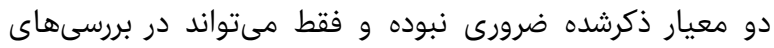

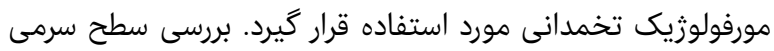
هورمون آنتىمولرين نيز براى تشخيص PCOS معيد مورديار مناسبى 
جدول \&) ضرايب همبستكى يِيرسون بين متغيرهاى كمى (استراديول، برولاكتين،

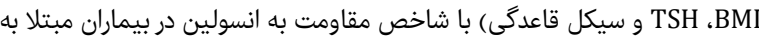

PCOS

\begin{tabular}{|c|c|c|c|c|}
\hline \multicolumn{2}{|c|}{ انسولين r ساعت بعد از غذا } & \multicolumn{2}{|c|}{ انسولين ناشتا } & \multirow{3}{*}{ متغيرها } \\
\hline سطح & ضريب & سطح & ضريب & \\
\hline معنىدارى & همبستخى & معنىدارى & همبستخى & \\
\hline.$/ 11 \mathrm{~V}$ & $.1 .9 k$ &.$/ 4$ & $\% \cdot v k$ & TSH \\
\hline$\cdot 1 \cdot V k$ & $-\bullet / 11$ & $\bullet \bullet \Delta$ & -.1199 & برولاكتين \\
\hline.$/ . .1$ & $\cdot / r \cdot r$ &.$/ .0 k$ &.$/ 94 q$ & سيكل قاعدىى \\
\hline.$/ .1$ &.$/ 198$ &.$|\cdots|$ &.$/$ mek & BMI \\
\hline$\cdot / V 1 Q$ & $.1 . r r$ & . $V \Delta Q q$ & -.1 .19 & استرادبول \\
\hline
\end{tabular}

بحث

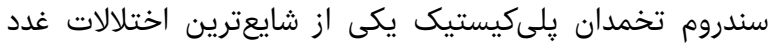

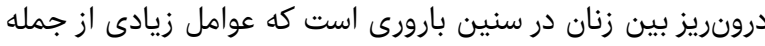

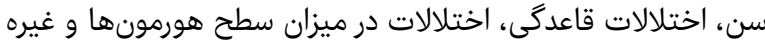

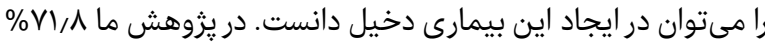

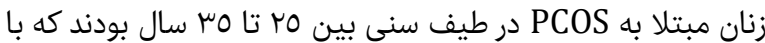

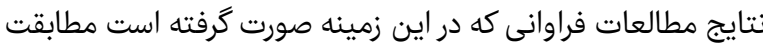

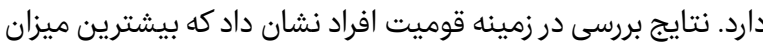

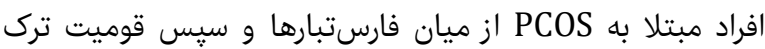

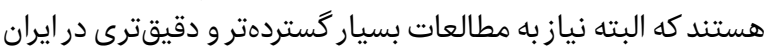

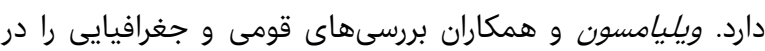

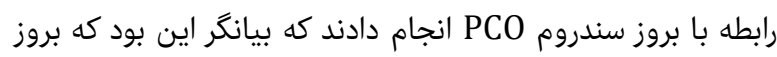

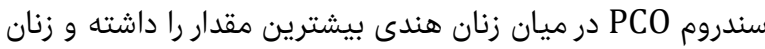

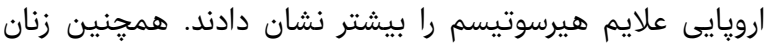

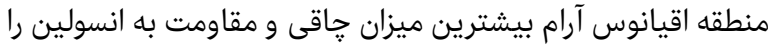

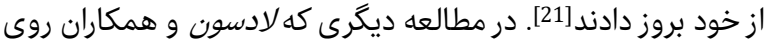

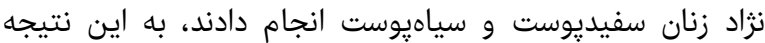

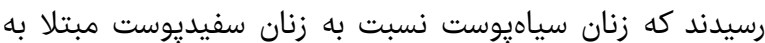
PCOS

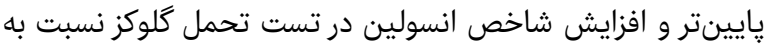

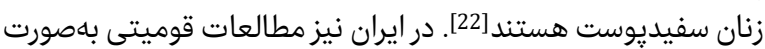

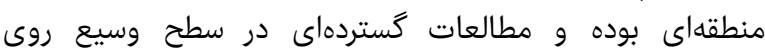
قوميتهاى مختلف صورت نكرفته است.

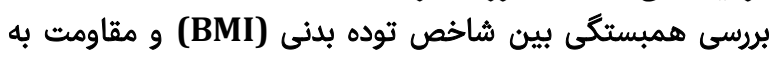

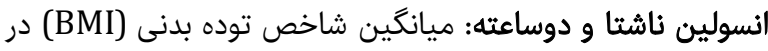

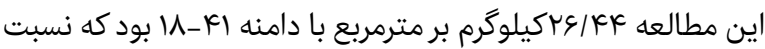

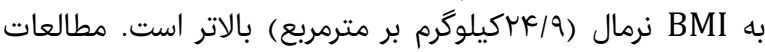
زيادى در زمينه ارتباط بين BMI و بروز

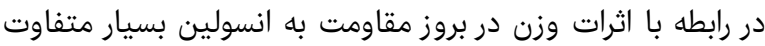

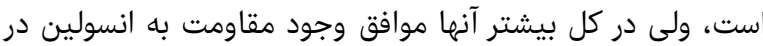

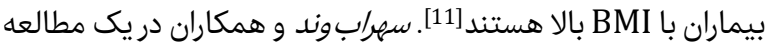

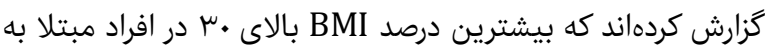

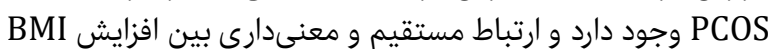

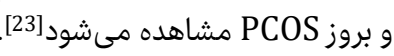

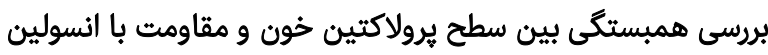

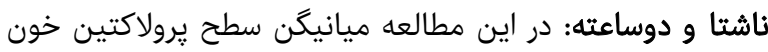

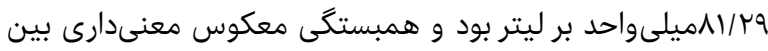

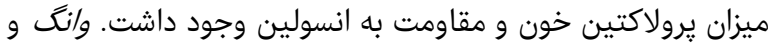

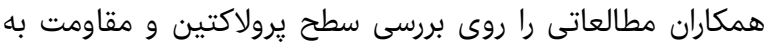

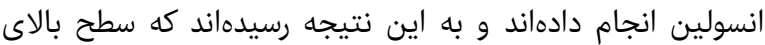
يرولاكتين، مقاومت به انسولين را در سلولهایى كبدى بدى كاهش

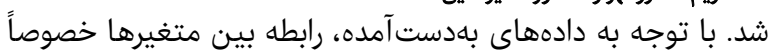

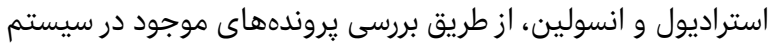

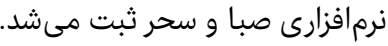

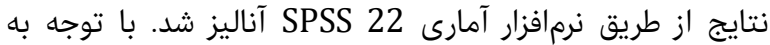

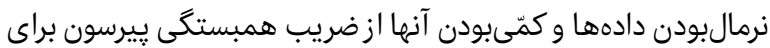

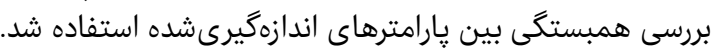

يافتهها

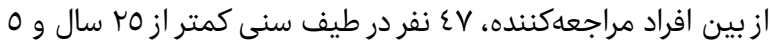

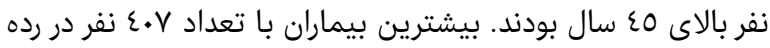

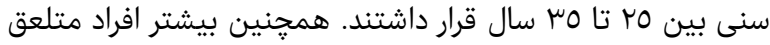
به قوميتهاى فارس و ترك بودند (جدول ؟ ب).

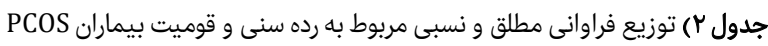

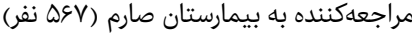

\begin{tabular}{|c|c|c|}
\hline درصد & تعداد & متغيرها \\
\hline & & رده سنى \\
\hline$\Lambda / \mu$ & FV & كمتر از ه سال \\
\hline$V / / \wedge$ & $k \cdot V$ & \\
\hline $19 /$ & $1 \cdot 1$ & 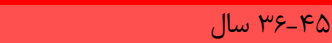 \\
\hline.$/ 9$ & Q & بيشتر از FQ سال \\
\hline & & قوميت \\
\hline$k \cdot 18$ & $r \mu$. & فارس \\
\hline rq/^ & 199 & ترك \\
\hline$V / \varepsilon$ & $k \mu$ & كرد \\
\hline$F / 8$ & re & كيلك \\
\hline$r / \Lambda$ & 19 & تركمن \\
\hline$r / \mu$ & Ir & لر \\
\hline $1 / 1$ & 1. & عرب \\
\hline.$/ 1$ & 1 & افغان \\
\hline $1 . / 4$ & $\Delta 9$ & ساير قوميتها \\
\hline
\end{tabular}

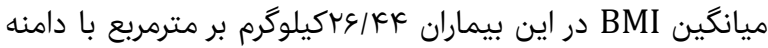

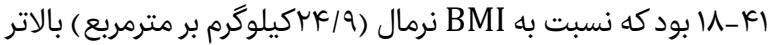

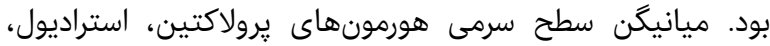

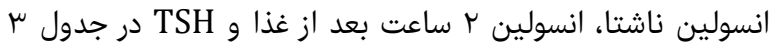

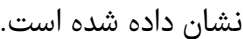

جدول س) ميانكَين آمارى متغيرهاى مورد مطالعه در افراد مبتلا به PCOS

\begin{tabular}{|c|c|}
\hline ميانكين آمارى & متغيرها \\
\hline$r T / \varepsilon \varepsilon \pm \varepsilon / 11$ & (كيلو Bرم بر مترمربع) BMI \\
\hline$\left|\mu^{\mu} / \mu\right| \cdot / 1 \Lambda$ & انسولين ناشتا (ميلىواحد بر ليتر) \\
\hline $07 / \cdot \cdot \pm 0 \varepsilon / 17$ & انسولين rاساعته (ميلىواحد بر ليتر) \\
\hline$r / 00 \pm 1 / V \varepsilon$ & TSH (ميلىواحد بر ليتر) TSH \\
\hline$\Lambda 1 / r q \pm 170 / \mu \mathrm{r}$ & يرولاكتين (ميلىواحد بر ليتر) \\
\hline $7 r / 7 \Lambda \pm 7 \cdot / \varepsilon 7$ & استراديول (ييكوگرم بر ميلىليتر) \\
\hline $1 / 97 \pm 1 / .7$ & سيكل قاعدگى (ماه) \\
\hline
\end{tabular}

بين ميزان BMI و افزايش انسولين ناشتا و دوساعته همبستگى

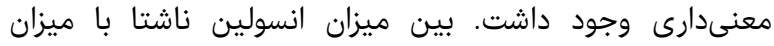

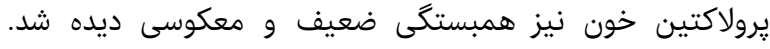

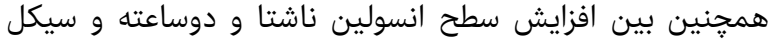

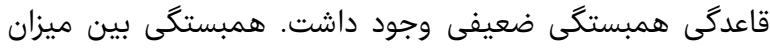

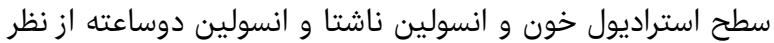

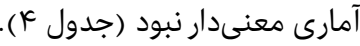




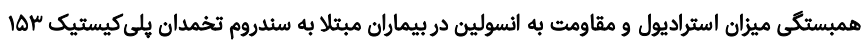

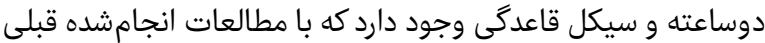
نيز مطابقت دارد. اخرجه افزايش مقاومت به دارد انسولين دربيش ازنيمى از افراد مبتلا به ديه

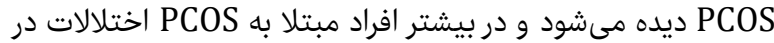

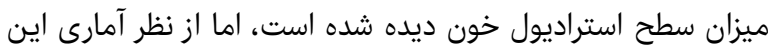

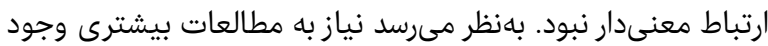

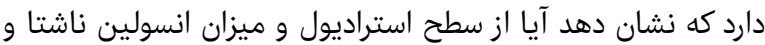

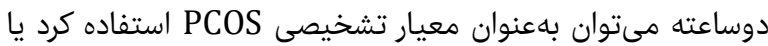

\section{نتيجه گيرى}

بين سطح استراديول خون و ميزان مقاومت به انسولين همبستگى ندا

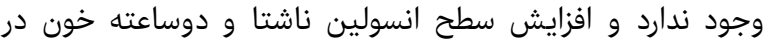

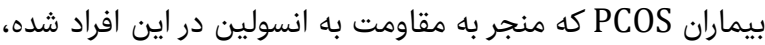

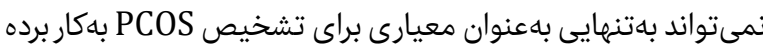

تشكر و قدردانى: از كليه همكاران كلينيك نابارورى بيمارستان

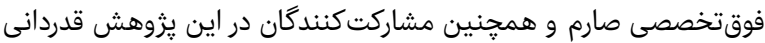

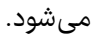
تاييديه اخلاقى: اين يزوهش بهر به تاييد كميته اخلاق دانشكاه علوم يزشكى

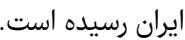

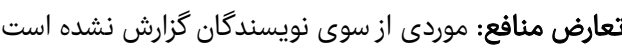

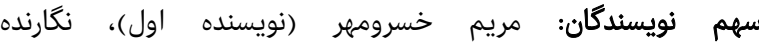

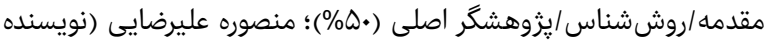

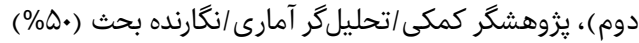

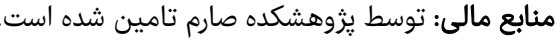

منابع

1- Kachoie A, Jahanipoor A, Ghahiri A. Evaluation of serum prolactin levels in patients with polycystic ovary syndrome. J Isfahan Med School. 2012;30(182):353-8. [Persian]

2- Balen AH. Polycystic ovary syndrome (PCOS). Obstet Gynaecol. 2017;19(2):119-29.

3- Baldani DP, Skrgatic L, Ougouag R. Polycystic ovary syndrome: Important underrecognised cardiometabolic risk factor in reproductive-age women. Int J Endocrinol. 2015;2015:786362.

4- Welt CK, Carmina E. Lifecycle of polycystic ovary syndrome (PCOS): From in utero to menopause. J Clin Endocrinol Metab. 2013;98(12):4629-38.

5- Sirmans SM, Pate KA. Epidemiology, diagnosis, and management of polycystic ovary syndrome. Clin Epidemiol. 2014;6:1-13.

6- Legro RS, Arslanian SA, Ehrmann DA, Hoeger KM, Murad MH, Pasquali R, et al. Diagnosis and treatment of polycystic ovary syndrome: an Endocrine Society clinical practice guideline. J Clin Endocrinol Metab. 2013;98(12):4565-92.

7- McLuskie I, Newth A. New diagnosis of polycystic ovary syndrome. BMJ. 2017;356:i6456.

8- Barber TM, Dimitriadis GK, Andreou A, Franks S. Polycystic ovary syndrome: Insight into pathogenesis and a common association with insulin resistance. Clin Med. 2015;15(Suppl 6):s72-6.

9- Jelodar G, Askari K. Effect of Vitex agnus-castus fruits hydroalcoholic extract on sex hormones in rat with induced polycystic ovary syndrome (PCOS). Physiol
مى دهد و بهطور معنىدارى با كاهش شيوع ديابت و كاهش مقاومت

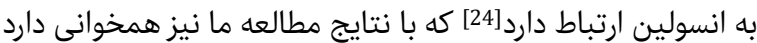

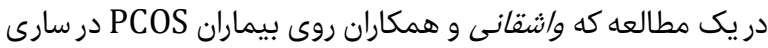

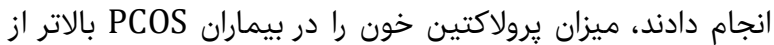

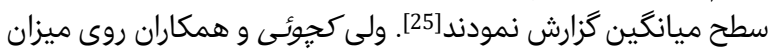

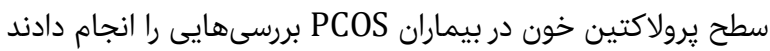

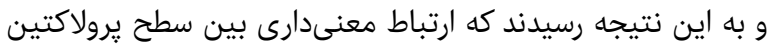

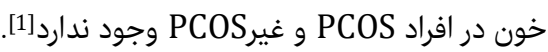

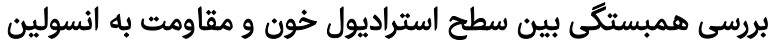

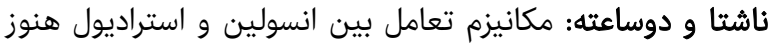

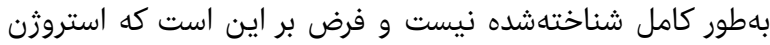

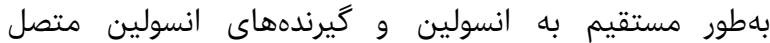

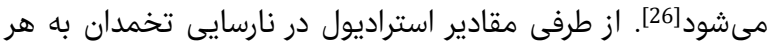

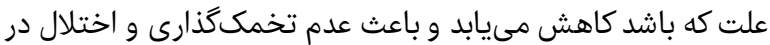

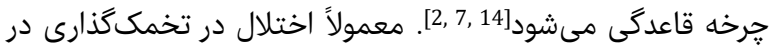

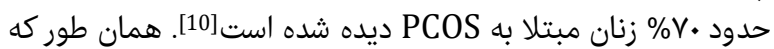

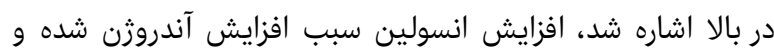

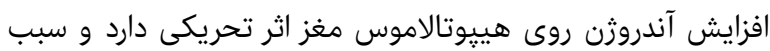

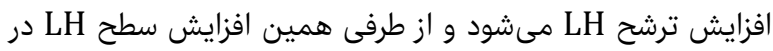

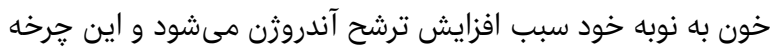

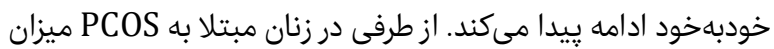

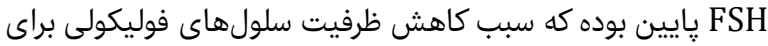

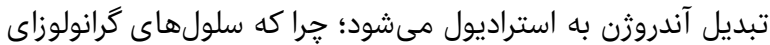

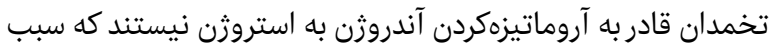

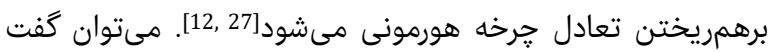

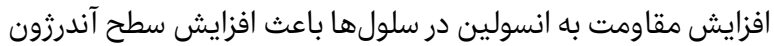

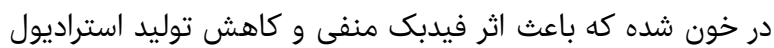

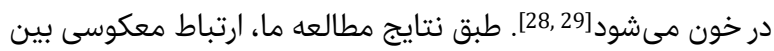

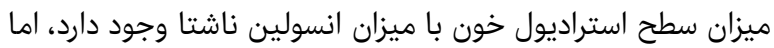

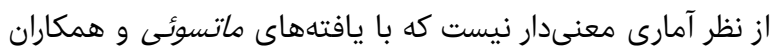

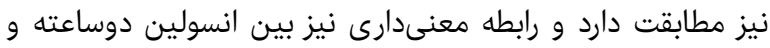
استراديول وجود ندارد [30].

بررسى همبستگى بين ميزان سطح TSH با ميزان مقاومت ندارد به به

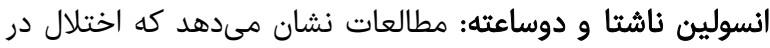

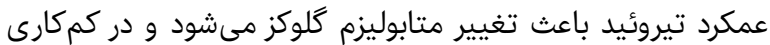

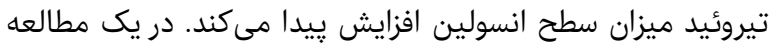

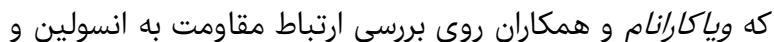

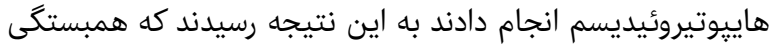

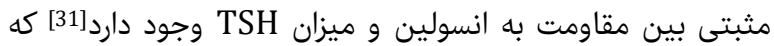

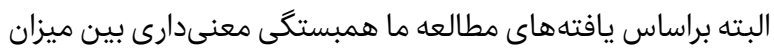

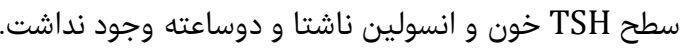

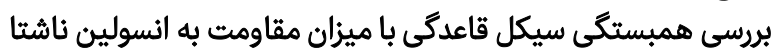

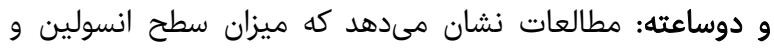

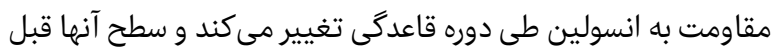

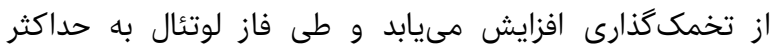

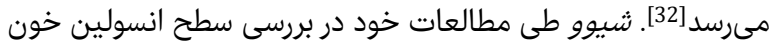

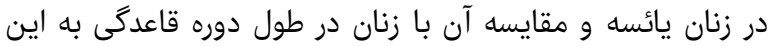

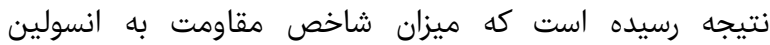

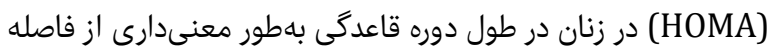

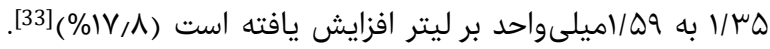

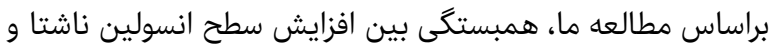


impact of ethnicity on the presentation of polycystic ovarian syndrome. Aust N Z J Obstet Gynaecol. 2001;41(2):202-6.

22- Ladson G, Dodson WC, Sweet SD, Archibong AE, Kunselman AR, Demers LM, et al. Racial influence on the polycystic ovary syndrome phenotype: A black and white case-control study. Fertil Sterili. 2011;96(1):224-9. e2. 23- Sohrabvand F, Ansari S, Bagheri M. Efficacy of combined metformin-letrozole in comparison with metformin-clomiphene citrate in clomiphene-resistant infertile women with polycystic ovarian disease. Hum Reprod. 2006;21(6):1432-5.

24- Wang T, Lu J, Xu Y, Li M, Sun J, Zhang J, et al. Circulating prolactin associates with diabetes and impaired glucose regulation: a population-based study. Diabetes Care. 2013;36(7):1974-80.

25- Vasheghani F, Jafari G, Khan Ahmadi M. Polycystic ovarian disease in the females 15-45 years old referring to endocrine and gynaecology clinic of Imam khomeini hospital of sari township 2000-2001. J Mazandaran Univ Med Sci. 2002;12(36):52-9.

26- Saremi A. Reproductive Medicine. Tehran: Sarem; 2016. [Persian]

27- Shabani Azim F, Dashti N. Review of Clinical Manifestations and Long-Term Outcomes of Polycystic Ovary Syndrome (PCOS). J Lab Q Diagn. 2017;9(36):1927. [Persian]

28- Vehkavaara S, Westerbacka J, Hakala-Ala-Pietilä T, Virkamäki A, Hovatta O, Yki-Järvinen H. Effectof estrogen replacement therapy on insulin sensitivity of glucose metabolism and preresistance and resistance vessel function in healthy postmenopausal women. J Clin Endocrinol Metab. 2000;85(12):4663-70.

29- Shafie SM, Hilf R. Relationship between insulin and estrogen binding to growth response in 7, 12dimethylbenz (a) anthracene-induced rat mammary tumors. Cancer Res 1978;38(3):759-64.

30- Matsui S, Yasui T, Tani A, Kunimi K, Uemura H, Yamamoto $\mathrm{S}$, et al. Associations ofestrogen and testosterone with insulin resistance in pre-and postmenopausal women with and without hormone therapy. Int J Endocrinol Metab. 2013;11(2):65-70.

31- Vyakaranam S, Vanaparthy S, Nori S, Palarapu S, Bhongir AV. Study of insulin resistance in subclinical hypothyroidism. Int J Health Sci Res. 2014;4(9):147-53.

32- Yeung EH, Zhang C, Mumford SL, Ye A, Trevisan M, Chen $\mathrm{L}$, et al. Longitudinal study of insulin resistance and sex hormones overthe menstrual cycle: the BioCycle Study. J Clin Endocrinol Metab. 2010;95(12):5435-42.

33- Sheu WH-H. Alteration of insulin sensitivity by sex hormones during the menstrual cycle. J Diabetes Investig. 2011;2(4):258-9.
Pharmacol. 2012;16(1):62-9.

10- Richardson MR. Current perspectives in polycystic ovary syndrome. Am Fam Physician. 2003;68(4):697704.

11- Kawwass JF, Loucks TL, Berga SL. An algorithm for treatment of infertile women with polycystic ovary syndrome. Middle East Fertil Soc J. 2010;15(4):231-9.

12- Villella S, Trickey R. Polycystic Ovary Syndrome. J Complement Med CM. 2004;3(4):78.

13- Ahmadi A, Mostafavi M. Effect of licorice root hydroalcoholic extract to improve the quality of fertility in hyperandrogenism-induced polycystic ovary syndrome by letrozole in mice. J Shahrekord Univ Med Sci. 2015;17:1-12. [Persian]

14- Goodman NF, Cobin RH, Futterweit W, Glueck JS, Legro RS, Carmina E, et al. american association of clinical endocrinologists, American college of endocrinology, and androgen excess and PCOS society disease state clinical review: Guide to the best practices in the evaluation and treatment of polycystic ovary syndrome-part 1 . Endocr Pract. 2015;21(11):1291-300.

15- Ghafurniyan H, Azarnia M, Nabiuni M, Karimzadeh L. The effect of green tea extract on reproductive improvement in estradiol valerate-induced polycystic ovary polycystic ovarian syndrome in rat. Iran J Pharm Res. 2015;14(4):1215-23.

16- Ruta DK, Kirtimalini S, Rahul K, Mrudula K, Dimple C. Contemporary and traditional perspectives of polycystic ovarian syndrome (PCOS): A critical review. IOSR J Dent Med Sci. 2014;13(9):2279-861.

17- Teede HJ, Misso ML, Costello MF, Dokras A, Laven J, Moran L, et al. International evidence-based guideline for the assessment and management of polycystic ovary syndrome [Internet]. Australia: Australian Clinical Practice Guidelines; 2018 [Cited 2018 May 10]. Available From:

https://www.clinicalguidelines.gov.au/portal/2598/inte rnational-evidence-based-guideline-assessment-andmanagement-polycystic-ovary

18- Rosenfield RL. The diagnosis of polycystic ovary syndrome in adolescents. Pediatrics. 2015;136(6):115465.

19- Zafari Zangeneh F, Minaee B, Amirzargar A, Ahangarpour A, Mousavizadeh K. Effects of chamomile extract on biochemical and clinical parameters in a rat model of polycystic ovary syndrome. J Reprod Infertil. 2010;11(3):169-74.

20- Bijeh N, Delpasand A, Hejazi K. The effect of different intensities of aerobic exercise on insulin levels and insulin resistance in rats with polycystic ovary syndrome. Q J Sabzevar Univ Med Sci. 2014;21(6):1163-72. [Persian] 21- Williamson K, Gunn AJ, Johnson N, Milsom SR. The 